In none of the ulcers on which I have operated by simple gastro-enterostomy have we found anything in the "followup " to suggest the subsequent development of cancer. Further, as will be seen from the tables, all large ulcers and many of the smaller have been excised-sometimes by themselves, at other times as part of the stomach removed in partial gastrectomy. I have not kept all measurements, but in a good proportion of the cases the ulcer was the size of a five-shilling piece or half-crown (see Fig. 7), without any

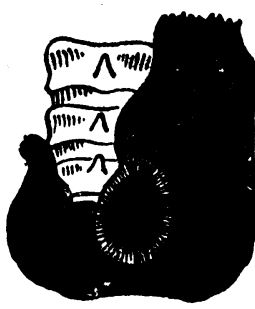
histological evidence of malignancy and without subsequent recurrence. On the other hand, all malignant ulcers which I have encountered have been of the size of a five-shilling piece and upwards.

In no case of ulcer, large or small, has cancer subsequently developed where the histological findings on the specimen removed at operation were non-malignant.

The immediate mortality in the FIG. 7:-Drawing from Case No. 137. An exnon-malignant ulcer of the posterior wall, erod ing pancreas, and transverse mesocolon. various groups is almost entirely due to pulmonary complications. To what extent are these pulmonary complications the result of the anaesthetic? The matter has been debated many times. Personally I think the anaesthetic is usually responsible. I have not had an opportunity of using ethylene, which American surgeons find such a help. The danger of pushing anaesthesia to the point of complete relaxation, and the consequent difficulty in ciosing an incompletely relaxed abdomen, have been held responsible for many of the ventral hernias in the upper abdomen. Insomuch as this complication is common to all abdominal operations $I$ have not included cases of post-operative hernia as unsatisfactory results in the figures above given.

In the choice of a site for the abdominal incision which will avoid ventral hernia surgical opinion is swinging back to the mid-line, where the interlacing fibres give a secure grip to the sutures. Personally I like to reinforce the continuous catgut in the essential layer with " figure of 8 " silkworm-gut sutures held taut on a frame. Since adopting this method I have so far had no ventral hernia in any gastric or duodenal case, although many times the condition of the patient did not warrant pushing the anaesthetic to complete relaxation, and in some cases, especially in perforation; the wound did not heal without some suppuration.

\section{TUMOURS OF THE FRONTAL LOBE OF THE BRAIN.}

BY

J. PURDON MARTIN, M.A., M.D., M.R.C.P. ASSISTANT PHYSICIAN TO THE NATIONAL HOSPITAL FOR NERVOUS DISEASES, QUEEN SQUARE; PHYSICIAN TO THE SEAMEN'S AND BOLINGBROKE HOSPITALS.

Tumouns of the frontal lobes of the brain anterior to the pre-central gyrus are notoriously difficult to diagnose. The following three cases in which the necropsies were performed within a period of less than three months seem to be worth recording because they form a group in which there was considerable similarity, and they indicate the chief lines of a general clinical picture.

Case I.

An engineer, admitted to the National Hospital, Queen Square had felt perfectly well up till March, 1926; one morning in that month, after walking a few yards from his house, he ran back, mounted the stairs two at a time, stooped to pick up a book, and fell unconscious. When his wife reached the room a few moments later he was still unconscious, was foaming at the mouth, and had bitten. his tongue; his face and. jaw were twitching, but not his limbs, and there was no incontinence. It is said that the attack lasted ten to fifteen minutes; the patient did not then recover consciousness, but appeared to pass into a slcep, from which he could not be roused. After several hours of sleep he had another fit. described as "most violent," in which he "struggled" with all his limbs. After a few hour's' more sleep he recovered consciousness and complained of severe headache, but nothing abnormal was noticed about him; his mental condition was that of a patient who had had a severe illness, and he was content to commit himself unreservedly to the care of others. Subjectively the patient recovered considerably in a few days, but his mental state of docility persisted more or less. He returned to his work and seems to have performed it normally for three months; at the end of that period he had another fit. This, like the former, was a generalized seizure with unconsciousness : after this he did not return to work. For the next six months he was free from gross symptoms, but there was some slight mental deterioration; he lost his initiative, relied increasingly on his wife, and became so forgetful and absent-minded that he could not be trusted to go out alone. He does not seem to have been troubled with headaches during this period. On Christmas Day, 1926, he had a severe headache; this persisted throughout the next day and led up to another fit in the afternoon of December 27th. My first The patient then had had continuous headache, not, however, of any great intensity, since the occurrence of the last fit.

This examination in January, except for a certain abnormal This examination in January, except for a certain abnormal
mental - state and two objective signs to be mentioned later, mental state and two objective signs to be mentioned later,
revealed nothing abnormal. No defect could be discovered in the revealed nothing abnormal. No defect could be discovered in the
motor or sensory functions; in particular, the optic discs were normal, as also were the visual acuity and the visual fields. The sense of smelt was intact in both nostrils, and odours could be distinguished and named. The cerebro-spinal fluid showed no cells and no excess of protein; the Wassermann reaction was negative both in the fluid and blood. An $x$-ray examination of the skull gave no indication of any bony change. The two slight signs referred to were an occasional fine tremor of the hands, such as is comto were an occasional fine tremor of the hands, such as is com-
monly seen in nervous patients at a first examination, and an monly seen in nervous patients at a first examination, and an
exaggerated flexor response in the plantar reflexes. The patient exaggerated flexor response in the plantar reflexes. The patient
remained in hospital for four weeks, and during that time had frequent headaches, but after the lumbar puncture the headache ceased for several dajs. After investigation he was allowed to go home (he said that he " felt quite well"), and was instructed to luminal were given during this time. After two months the patient returned to the out-patient department, complaining of a patient returned to the out-patient department, complaining of a loss of vision and the occurrence of "petit mal" attackswas found a week later that the visual acuity of the left eye had diminished to $6 / 60$ and that of the right to $6 / 9$. The left disc showed some doubtful pallor of its temporal half and the right appeared normal; colours of small objects could not be distinguished in any part of the field of the left eye.

The patient was again admitted to hospital under my care on April 9th, 1927. At that time, more than a year after his first fit, his condition was as follows. He answered questions and behaved intelligently, and had no delusions or hallucinations, but he did intelligently, and had no delusions or hallucinations, but he did
not. seem to realize fully the seriousness of the threatened blindnot. seem to realize fully the seriousness of the threatened blindness. He was probably somewhat deficient emotionally, and was
slightly euphoric : there was less than the usual emotional modulation in his voice. His speech was tremulous and slow, but there was no aphasia; his memory was bad. The sense of smell was
still normal on both sides. The vision was seriously impaired. In the right eye there was a central scotoma which was quickly enlarging, though the disc appeared normal. In the left eye acuity was reduced to counting fingers at half a metre, and the central and upper temporal parts of the field were completely central and upper temporal parts of the field were completely obliterated; on the disc there was some temporal pallor. The
pupil reactions and eye movements were normal. The right palpupil reactions and eye movements were normal. The right palof the right facial muscles, but such weakness as there was did not affect voluntary movements. The remaining cranial nerve functions were normal. In the limbs no motor or sensory abnormality was found except that the patient was at times generally tremulous. This. was a fine irregular tremor, not unlike that seen in early stages of general paralysis, and it affected his The plantar reflexes were peculiar. in that they were very briskly The plantar reflexes were peculiar in that they were very briskly
flexor: the slightest touch of the sole produced full and immediate flexor: the slightest touch of the sole produced full and immediate
flexion of the great toe, unaccompanied by any withdrawal of the foot. The gait was normal and there was no incontinence.

During. May the vision rapidly deteriorated and the -temporal parts of both discs became pale; the visual field of the right eye was almost entirely obliterated by enlargement of the central scotoma. An $x$-ray photograph of the skull now showed some change about the pituitary fossa, and rather open sutures change about the pituitary fossa, and rather open sutures suggesting intracranial pressure. It seemed that there must be a
tumour involving the optic chiasma and the optic nerves, affecting tumour involving the optic chiasma and the optic nerves, affecting region by operation. On June 22nd the operation was performed by. Mr. Sargent, the exploration being made through a quadrilateral opening in the left frontal bone. Part of the temporal bone in the temporal fossa was extremely thin; the dura was very tense, and to relieve the tension about $40 \mathrm{c.cm}$. of cerebrospinal fluid were withdrawn by lumbar puncture. When the dura had been opened-and the left frontal lobe was elevated a soft gelatinous-looking mass was seen protruding from under the frontal lobe, on the outer side of the olfactory bulb. It surrounded the left optic nerve and the chiasma, and from these structures a portion of it was dissected off; the site of origin of this growth could not be determined. This operation resulted in a considerable improvement in the patient's vision, and the wound healed uneventfully.

A few weeks later definite weakness of the right side of the face and right arm began to develop and was accompanied by motor aphasia. At this stage (July 29th, 1927) the plantar reflexes were still of the same exaggerated flexor type as before, and
stroking of the sole of the left foot caused flexion of the great toe on the right side as well as on the left; but by Oppenheim's method a feeble extensor response could be obtained on the left During the last few weeks of his life the patient was emotional and very depressed. His gen

At the necropsy a very hard tumour was found on the medial 
surface of the left frontal lobe; it reached the inferior surface of the lobe in the region of the olfactory tract and passed backwards to, but did not involve, the optic chiasma. The anterior clinoid processes were completely eroded, so that there was a hollow in front of the pituitary body. When the brain was cut, after hardening, the left frontal lobe was found to be filled with a firm whitish tumour-mass, which shaded off imperceptibly into the white matter of the brain; it involved the head of the caudate nucleus, and, medial to that, formed a rounded boss on the internal aspect of the lateral ventricle. It did not extend higher than the genu of the corpus callosum. Microscopically it was nuclei (astrocytoma fibrillare).

Summary.

Onset of the symptoms with a severe convulsive attack; slight but increasing mental changes of the nature of a loss of initiative, loss of memory, increasing reliance on others, insufficien realization of his condition, euphoria; tremor of the hands and in the voice, not constant and not noticed when the patient was, in bed; headaches; further convulsions and frequent " petit mal" attacks; a year after the onset rapid diminution of vision and exaggerated briskness.

The necropsy confirmed the presence of a tumour in the left frontal lobe. A noteworthy negative feature was the absence until the last stages of the illness of practically all abnormal motor phenomena except tremor; there were no symptoms in the olfactory system, no speech symptoms, and no ataxia.

\section{CASE II.}

An electrician, aged 34 , seen by me at the London Temperance Hospital, had been employed in Greece, where it was his duty to instruct and supervise native workers. Seven months before his admission to hospital he had begun to make stupid mistakes and had become useless as a supervisor; he was forgetful, and and had become useless as a supervisor; he was forgetful, and before admission he began to suffer from headaches; he referred the pain chiefly to the right mastoid region, where he had had an injury some years previously. He had never had a fit. There was no history of vomiting. At times he was excessively drowsy.

In giving his history he made mistakes in dates and in periods of time. When I examined him on October 11th, 1927, he did not know the date, and had not even an approximate idea of the length of time he had been in hospital. He was slightly euphoric. $\mathrm{He}$ seemed to have little understanding of discipline, and broke the rules of the hospital in regard to smoking, but evidently without any malice or appreciation of wrong-doing, or even of the fact that he was doing anything unusual. He was, in fact, very submissive when addressed. Sometimes during examination he would do something other than he was asked, apparently because he failed to form a correct idea of the action which was required of him (ideational apraxia). Repeatedly when asked to show his teeth he put out his tongue, and he did not realize his show his teeth he put out his tongue, and he did not realize his and deseribe what he had read, and could write.

There was no loss of smell. The optic discs may have been a little blurred in the upper nasal quadrants, but were not definitely pathological; the visual fields responded normally to finger tests, and the pupils and,eye movements were normal. There was a slight " smoothness" or emotional weakness of the left side of the face at rest, but no weakness was seen in voluntary movement. The remaining cranial nerve functions were normal. In the arms power, sensation, tendon-jerks, pointing tests, and the control of fine movements were all normal; there was no persisting hand-grasp. While the patient was lying down there was usually no tremor of the hands, but when he sat up there were frequent short attacks of gross irregular tremor like that of general paralysis, more marked, if anything, in the left hand than in the right. All the abdominal reflexes were brisk. In the lower limbs power, sensation; and tendon-jerks were normal. The plantar reflexes were both briskly flexor in an extreme degree, the merest touch of the sole causing full and immediate flexion, without any withdrawal of the foot such as occurs when the-sole is very sensitive; with persistent stimulation the flexor response on the lèft side seemed to tire, and gave place to a slight extensor reflex, with some withdrawal at the knee. When the patient stood up he tended to fall backwards, and when walking he was
inclined to deviate to the right. He had incontinence (of the mental type) of both urine and faeces. The cerebro-spinal fluid was normal in its cellulur and albumin content, and the Wassermann reaction was negative both in this fluid and in the blood. There was a slight irregular rise of temperature, and the pulse There was a slight irregular rise of temperature, and the pulse expressed that the cause of the symptoms was a tumour, or
possibly chronic absesses, involving both frontal lobes, but possibly chronic

On October 20 th the patient had a convulsion, and after several hours of unconsciousness he died. The necropsy was performed at the hospital by Dr. Sanguinetti. In the horizontal section of the brain, passing through the genu of the corpus callosum, there was a tumour on the right side as large as a pigeon's egg, just anterior to and touching the caudate nucleus. It lay right in the path of the fibres of the "forceps minor", radiating out into path of the fibres of the "forceps minor" radiating out into was well demarcated, but it extended downwards and forwards into the lobe, and there its boundary was less definite. On the left side there was a mass of tumour, probably of later development, occupying the medial part of the frontal lobe just anterior to the corpus callosum; its outline was much less definite than that of the tumour on the right side. Microscopically the growth was a polymorphocellular sarcuma.
Gradual development Summary

"Gradual development of mental symptoms-loss of mental " sharpness," stupid mistakes, inability to supervise others, loss of memory, later ideational apraxia; headaches; tremor of the hands like that in general paralysis of the insane; tendency to fall backwards when standing and to deviate to the right when facial , excessively bis a the left side with persistent stimulation.

The presence of a bilateral frontal lobe tumour was verified after death. The absence of definite optic disc changes and of vomiting, of olfactory symptoms, aphasia, and all kinds of paralysis should be noted.

CASE III.
An unmarried woman, aged 58, a boxmaker, came to the outpatients' department at the National Hospital, Queen Square, on November 1st, 1927; she was said to have suffered from depression for about a year, but until July 13 th she was otherwise well. On that day she had a fit, without warning, in the street. After this she was " not quite herself," though she returned to her work and seems to have carried it on normally. During this period it was observed that she often rubbed her nose as if it itched. She remained at work until September 18th, when she was moro depressed than usually, and during the afternoon she fell down
in a fit. This was the first of a series of fits which lasted t:1l in a fit. This was the first of a series of fits which lasted till
11 o'clock the next morning, and the patient was more or less 11 o'clock the next morning, and the patient was more or less
unconscious for forty-eight hours. Early in the attack she was removed to a hospital; when she recovered consciousness she had no paralysis or aphasia, and she left the hospital after a stay of five days. She was able to dress herself and walk to the car which took her home. After her return her manner was strange; she was " absent-minded" and confused, her memory was bad, and she could not be trusted to do ordinary things, such as to dress herself, because she would make mistakes; she had to be directed constantly and was very docile. About a month later she began to "worry about, things which she ought to have known were perfectly all right:" She had frequent headaches and sometimes complained of pain over the right eye.

When $I$ addressed a question to her at the out-patient department she did not answer, but turned to her companion as anwer and some of her complaints correctly. Only in answer to one quesand some of her complaints correctly. Only in answer to one ques-
tion regarding the duration of a symptom did she say, ". I don't tion regarding the duration of a symptom did she' say, "I don't remember." Her speech was somewhat slow and monotonous, but malities in the cranial nerve functions or any changes in rowe or sensation in the limbs. The tendon-jerks were brisk, the right plantar reflex flexor, and the left doubtfully extensor. There was a general rhythmical tremor of the hands, and the patient volunteered the statement, which was confirmed by observation, that the tremor was more pronounced in the left hand than in the the tremor was more pronounced in the left hand than in the right; the difference was, however, variable. All the abdominal
reflexes were weak. The, provisional diagnosis was "frontal tumour, right or bilateral."

She was admitted to hospital under my care two days later. Her general condition quickly became worse; she was very drowsy, tended to become comatose, and was incontinent of urine and $(300 \mathrm{~mm}$. aq.); it contained a very large number of cells (270 per mm.), of which nearly all were polymorphonuclears, and it had a very high protein content (0.130 per cent.). This cerehro spinal fluid analysis suggested the presence of an abscess, but several other features of the case were opposed to that view. However, in case an abscess might be present, and in the hope of relieving the patient's general condition by cerebral decompression, it was decided to operate. On November 8th Mr. Armour exposed the right frontal lobe by a fronto-temporal craniectomy; in the posterior portion of the second frontal convolution there was a small brownish tumour, about half an inch in diameter, slightly raised above the surface of the neighbouring convolutions, and this was removed. After the operation the patient remained in a very drowsy and feeble state for three days and then died.

At the necropsy, whẹn the brain had been hardened, section revealed no other mass in the right frontal lobe, but in the left frontal lobe there were several masses-similar to that which had the tip of the lobe and across the genu of the corpus callosum. The whole of the left frontal lobe was much larger than its fellow. Microscopic section of the mass removed at operation fellow. Microscopic section of the mass removed at operation
showed that it was a glioma. The masses in the left lobe had a showed that it was a glioma. The masses in the left lobe had a similar structure, and the remainder of the left frontal lobe was
filled with gliomatous tissue unusually rich in fibres. Such tissue would probably interfere little with the functions of the nerve cells of the part.

Summary.

A woman, aged 58 suffered from unusual mental depression for several months and then had a convulsive attack; after an interval of two months a very severe series of convulsions occurred. Mental changes then became apparent-confusion, loss of memory, liability to make mistakes in everyday duties, abnormal reliance on others headaches became frequent; tremor of the hands was observed. Towards the end the plantar responses had become not flexor, but indefinite.

After death a tumour was found involving both frontal lobes. The absence of signs ana symptoms in the olfactory and visual systems and the
are noteworthy. 
The difficulty in the diagnosis of frontal tumours lies in the paucity of the reliable physical signs and symptoms. Headache, vomiting, and papilloedema are still regarded as the cardinal signs of an established cerebral tumour, but in frontal lobe cases vomiting and papilloedema do not usually develop, and frontal tumours must be diagnosed in their absence. The absence of all recognizable paralysis and of aphasia has been noted in the foregoing cases. Only when the tumour lies towards the posterior part of the frontal lobe are such signs to be expected, the growth then being sufficiently near to the pre-central convolution, or to Broca's area or the insula, to interfere with the function of these parts by actual destruction or by oedema.

Generalized headache such as occurred in all three cases must be the result of increased intracranial pressure; it is therefore unlikely to occur until the growth is large enough to give rise to some dislocation of the braiu and consequent impediment to the outflow of cerebro-spinal fluid from the ventricles. In none of these cases was the general headache intense until other symptoms had been established. In Case III pain over the left eye was one of the patient's most frequent complaints; such local pains are probably due to a local stretching of the dura or to erosion of bone.

Convulsions were an early symptom in two out of the three cases, and were the first definite indication of disease. They occurred at a time when nothing abnormal was to be made out, except perhaps some vague mental change. They were generalized attacks, associated with prolonged headache, and sometimes with continued unconsciousness. Attacks of such severity and of such absolute suddenness are rarely caused by tumours in other parts of the brain. In one case typical " petit mal" attacks also occurred.

The mental alterations met with in frontal tumour cases are not specific for tumours of this localization; similar changes are common in cases of tumours in other parts of the brain if there has been prolonged intracranial hypertension; but when they are encountered in the absence of papilloedema these mental changes certainly have a localizing value. The most constant psychical modification-one which was met with in all these cases-is an abnormal submissireness or docility, and a child-like reliance on others; with this goes a loss of initiative. Some loss of memory is frequent, and confusion may occur: the patient may become liable to make mistakes and onissions in everyday actions, and in this way act indecently. The incontinence which occurred towards the end in two of these cases is to be regarded as a symptom of dementia and not as the result of any paralysis. The first patient of this group was somewhat euphoric; none of them showed that tendency to make a joke at every remark (Witzelsucht) which is probably more indicative of a frontal lobe lesion than any other single mental symptom. These mental changes occur with frontal tumours of either side of the brain.

Tremor was the only observed clinical sign except the mental changes which was common to all three of these cases. In the first patient it was a slight general tremulousness, which at first attracted little attention, but it was more pronounced when the patient was seen in the outpatient department than when he was at rest in the ward. I cannot say now whether it was greater on one side than on the other. In the second patient the tremor was most pronounced, and in his case it was a coarse tremor occurring in frequent short attacks each lasting a minute or so. These attacks seemed to be more likely to come on when he was sitting up or standing than when he was lying down; they usually affected both hands, but on one occasion, while in bed, he had a paroxysm of trembling affecting the left side only. The third patient's tremor, like that of the first, was a fine tremor, but it was more regular. It was more easily perceptible by palpation than by sight, and might easily have been overlooked; moreover, it was .bilateral. A tremor affecting the side of the body on which the tumour was situated was described in frontal lobe tumour cases by Dr. Grainger Stewart, and it is generally accepted that the tremor of these cases is homolateral. In two of my cases the tremor was bilateral, the tumour being bilateral also, but even in the third case, in which the tumour was confined to the left side, the tremor affected both sides in some degree. So far as I am aware there is no reason for referring this tremor to destruction of any particular part of the frontal lobes.

Reflex changes in all these cases were few. The tendon jerks were within normal limits; the abdominal reflexes were present in all, and in the first two were brisk. The plantar reflexes were indefinite in one case, but in the other two cases they were peculiarly exaggerated. The slightest touch to the sole of the foot caused full and immediate flexion of the great toe; the patients showed no signs of unusual sensitiveness of the sole of the foot and made no voluntary withdrawal or other movement. In both cases the phenomenon was bilateral, and in the first case, in which the tumour was on the left side, stimulation of the sole of the right foot caused brisk flexion of the great toe, not only on the right foot, but also on the left, the morement on the left side occurring a little later than that on the right. Stimulation of the left sole caused flexion of the left toe only. The exaggerated plantar response was one of the earliest abnormal signs in Case $\mathbf{I}$, being present at the time of my first examination of the patient, when no other abnormal physical signs were to be found. I have met this same phenomenon in two other cases in which there were meningeal tumours affecting the frontal lobes by pressure, but I am unable to say whether it is confined to cases of frontal lobe lesion. It is worthy of notice that while this phenomenon was actually present an extensor response could be obtained in Case $I$ by Oppenheim's method, and in Case II after persistent stimulation of the sole, the flexor response seeming to tire. The observation of the "crossed flexor response" in Case I was made by Dr. Ferguson, house-physician at the National Hospital. This peculiar sign could be obtained constantly from the time when it was first noticed up till the patient's death a month later; it is evidently a further development of the exaggerated flexor response, and its occurrence is evidence that the exaggerated flexor response is definitely a pathological sign.

Of the signs which may arise in the olfactory and visual systems little need be said. A unilateral anosnia is of more precise value than almost any other sign of frontal tumour, but unfortunately it rarely occurs early, and, in the first case described, it was entirely absent until other signs had put the diagnosis beyond doubt. Optic interference, if unilateral, is also very valuable, but bilateral visual defect such as occurred in the first of these cases has so many more common causes that it is likely to mislead.

The ataxia encountered in the second case is a wellknown sign in frontal tumours, but in my experience is only met with at an advanced stage of the disease, and both for this reason and on account of the difficulty of distinguishing it from the ataxia of a cerebellar lesion, it is of little practical value for diagnosis.

Differential Diagnosis.

Even when one is faced with definite clinical signs the diagnosis of these cases has to be made from general paralysis of the insane, cerebral syphilis, cerebral vascular disease, disseminated sclerosis, Schilder's disease, and other forms of gliosis, and also from tumours of the temporal lobe. The Wassermann reaction, the age of the patient, and the general state of the circulatory system may rule out the first three of these. Disseminated sclerosis is likely to cause more reflex changes than frontal tumour, while the other diffuse diseases are likely to be eliminated by the age of the patient or the rate of progress of the malady. The occurrence of severe generalized headaches is a point in favour of a neoplasm, and the cerebrospinal fluid pressures measured at lumbar puncture may give more precise evidence of its presence. Temporal lobe tumours usually bring about an homonymous visual field defect, they cause papilloedema relatively early, and they do not give rise to tremor. In the presence of merely the earlier signs of frontal tumour the most difficult point may be to determine on which side the tumour lies. The mental changes do not help to decide this, but the preponderance of tremor on one side indicates that the tumour is on the same side; any visual, elfactory, speech, or motor signis are likely to settle the point. The great tendency of frontal lobe tumours to become bilateral must be borne in mind. 
Summary.

Three cases of frontal lobe tumour are described, in two of which the growths were bilateral.

The only observed clinical signs which were common to all three cases were mental changes and tremor.

In two cases severe generalized convulsive attacks constituted the first definite symptom.

Attention is drawn to a peculiar exaggeration of the plantar reflexes encountered in two of the cases.

\section{TUMOUR OF THE BRAIN SIMULATING ENCEPHALITIS LETHARGICA.}

BY

\section{S. McCLEMENTS, M.R.C.S., L.R.C.P.}

THE rarity of a cerebral tumour giring rise to the clinical picture of encephalitis lethargica in its early and penultimate stages is so marked that the following case deserves attention.

A man, aged 57, had a mild attack of influenza in April, 1926, and was reported as completely well in the third week of the following month. Four weeks later an insidious onset of drowsiness and lethargy began, and was followed in a few days by dimness of vision for near objects. The drowsiness gradually vision; the temperature during the same period oscillated between $98^{\circ}$ and $100^{\circ} \mathrm{F}$. The period of stupor reached its height at the end of July, and was followed by marked improvement in the end of July, and was followed by marked improvement in the patient's condition, as shown by his taking an increasing interest
in his surroundings and in the disappearance of diplopia. Except for spasmodic twitching of his legs he had almost regained his normal health in September. In October and December, 1926, and April, 1927, he had relapses which were followed with marked improvement, athough it was noticed that each successive improvement left the patient a little more stuporous. In June, 1927, he became markedly lethargic, and on July 13th he was admitted to hospital for treatment, his condition having been definitely diagnosed by a neurologist as encephalitis lethargica. On admission his temperature was $98^{\circ} \mathrm{F}$., and his pulse 74 . He continued in a stuporous condition, but when awakened his mental condition appeared quite clear and he was able to answer questions in a appeared quite clear and he was able to answer questions in a
rational manner. The face was smoothed out and expressionless, the eyelids were in a condition of ptosis, his voice was monotonous, but not indistinct, his right eye showed an internal strabismus and on being questioned he said he saw two fingers instead of one which I held before him. There was dimness of vision for near objects, the pupillary reflex to light was absent on the right side and sluggish on the left; reaction to accommodation was normal. The tendon, abdominal, and plantar reflexes remained unaffected.

Although signs of improvement were occasionally manifested the stupor gradually deepened, and the vision of the right eye diminished so rapidly that a more detailed ophthalmological examination was made. The ocular reflexes had not altered since the previous examination; there was paresis of the external rectus on the right side, associated with well-marked papilloedema of the right optic disc, and the retinal veins of the left fundus appeared slightly swollen. These findings pointed to the probability of a neoplasm localized to the right frontal to the probabrain, producing pressure on the right second and sixth cranial brain, producing pressure on the righ

A cerebral gumma being suspected as the cause of the mischief a Wassermann test of the blood was performed; the reaction was positive. Antisyphilitic treatment, in the form of potassium iodide and liquor hydrarg perchlor. internally, and neosalvarsan int ramuscularly, was started at once, but there was no response, and the patient died on Ncvember 30th, 1927, eight weeks after the commencement of the treatment.

The autopsy revealed a spherical tumour, two inches in diameter and gelatinous in consistency, lying in a smooth walled cavity of the right frontal lobe of the cerebrum; posteriorly between the tumour and the wall of the cavity was a clot of blood an the tumour and the wall of the cavity was
inch long and a quarter of an inch broad.

The points of interest in the case are:

1. The earlier symptoms-namely, gradually progressing drowsiness, dimness of vision, diplopia, ptosis of eyelids, and occasional slight rises of temperature-were identical with the clinical manifestations in a large group of cases of encephalitis lethargica.

2. Complete absence of headache and vomiting, which are almost invariably present in the later stages of cerebral tumour.

3. The importance of ophthalmoscopic examination of the eyes in every case pointing to a lesion of the central nervous srstem.

It is very much regretted that there was no histological examination of the tumour; perhaps an expert in neuropathology would renture an opinion as to the nature of the neoplasm.

\section{ISOLATION OF B. TYPHOSUS FROM SEWAGE AND SHELLFISH. \\ BY}

W. JAMES WILSON, M.D., D.Sc.,

PROFESSOR OF HYGIENE AND PUBLIC HEALTH, QUEEN'S UNIVERSITY, BELFAST; WATER EXAMINER TO BELFAST WATER BOARD.

THE danger of consumption of sewage-contaminated water, shellfish, watercress, etc., is a cardinal article of the hygienist's faith, but hitherto it has not been possible to find objective evidence by the cultivation of the typhoid bacillus from the sewage. By the use of a new technique I can now demonstrate on an average the presence of one typhoid bacillus in each cubic centimetre of Belfast sewage.

Sir Alexander Houston ${ }^{1}$ in 1913 and 1914 examined 23,353 non-lactose fermenting colonies developing on plates inoculated mainly with samples of London (Barking) or Hendon sewage, and also with individual samples from Dublin, Belfast, Edinburgh, and Aberdeen. He was unsuccessful in isolating a single $B$. typhosus, and concluded that the typhoid bacillus is not uniformly present in $0.00066 \mathrm{c.cm}$. of crude sewage, and that this amount of sewage contains 704 excretal bacteria per cubic centimetre. Dealing with the difficulties of the task Houston writes:

"Sewage contains such an enormous number of bacteria that it is quite impracticable to examine more than a very small amount of this material. For example, to examine one cubic centimetre of crude sewage for typhoid bacilli by the direct plating method would mean making at least 1,000 special plate cultures and at least 20,000 primary subcultures, not to speak of the secondary and other cultures for the purpose of differentiation. It is, however, quite practicable to work with as much as $0.01 \mathrm{c.cm}$. of sewage spread over from 10 to 20 special plates, and therefore to make 200 to 400 primary cultures."

Non-lactose fermenting organisms are so common in sewage that many thousands or hundreds of thousands of such colonies would require to be examined in order to isolate a single typhoid bacillus. For example, in Belfast sewage I find that on an average 400,000 to 500,000 organisms develop from $1 \mathrm{c.cm}$. on MacConkey lactose bile salt agar plates, and that of these more than one-half are non-lactose fermenters. In such sewage I have found about one typhoid bacillus in each cubic centimetre, so that, using the MacConkey medium, there would have been a chance of isolating the bacillus if 250,000 non-lactose fermenting colonies had been tested. In all probability, even after such a Herculean effort, failure would have resulted, as the chances are that the typhoid bacillus would not have had a clear space on the plate to develop, and its growth would have been obscured and inhibited by the coliform colonies. The addition of brilliant green to the medium would doubtless render the isolation of $B$. typhosus from sewage not quite so difficult, but even so the chances against a non-lactose fermenting colony being composed of typhoid bacilli would be many thousands to one. In the examination of sewage for the presence of 13 . typhosus 1 therefore made use of the glucose-sulphite-iron-bismuthbrilliant green medium of Wilson and Blair, ${ }^{2}$ the efficiency of which depends on two original observations of mine: (1) B. typhosus in the presence of a fermentable carbohydrate is able to reduce a sulphite to a sulphide, and so to form a black colony in the presence of an iron salt. (2) Bismuth sulphite in the presence of a certain excess of sodium sulphite suppresses the growth of most coliform bacilli; in the presence of brilliant green the selective action is intensified. This medium has in my hands rendered the isolation of $B$. typhosus from enteric stools one of the easiest procedures in applied bacteriology, and recently by means of it B. A. Adams ${ }^{3}$ has isolated the same bacillus from the excreta of seagulls.

When 0.5 to $1 \mathrm{c.cm}$. of sewage is poured over the surface of a plate of the medium and allowed to dry, and is then incubated for twenty-four hours at $37^{\circ} \mathrm{C}$., black colonies with a metallic halo are developed, and also light green colonies of $B$. proteus. It is among the black colonies that $B$. typhosus is to be sought. All the black colonies, if subcultured on MacConkey or Endo medium, will be found to consist of non-lactose fermenters, so that these media are not very helpful in distinguishing between colonies of 7 . typhosus and those of other reducing bacteria. It was found that a very large proportion of the black 